\title{
Classification criteria for regular trees
}

\author{
KHANH NGUYEN
}

\begin{abstract}
We give characterizations for the parabolicity of regular trees.
Luokitusehtoja säännöllisille puille
\end{abstract}

Tiivistelmä. Esitämme säännöllisten puiden parabolisuudelle yhtäpitäviä ehtoja.

\section{Introduction}

Let us begin with the uniformization theorem of Klein, Koebe and Poincaré for Riemann surfaces. The celebrated theorem says that every simply connected Riemann surface $M$ is conformally equivalent (or bi-holomorphic) to one of three Riemann surfaces: the half plane $\mathbb{H}^{2}$ (surface of hyperbolic type), the complex plane $\mathbb{R}^{2}$ (surface of parabolic type), the Riemann sphere $\mathbb{S}$ (surface of elliptic type). Then $M$ admits a Riemannian metric $g$ with constant curvature. A simply connected Riemann surface is said to be hyperbolic if it is conformally equivalent to $\mathbb{H}^{2}$, otherwise we say that it is parabolic.

Let $M$ be a simply connected Riemann surface with Riemannian metric $g$. A $C^{2}$-smooth function $u$ defined in $M$ is superharmonic if

$$
-\Delta u \geq 0
$$

where $\Delta$ is the Laplace-Beltrami operator associated to the Riemannian metric $g$.

It is well known that every conformal mapping in dimension two preserves superharmonic functions (see [1, Page 135]). Since $\mathbb{H}^{2}$ possesses a nonconstant nonnegative superharmonic function and every nonnegative superharmonic function on $\mathbb{R}^{2}$ or $\mathbb{S}$ is constant, it then follows that there is no nonconstant nonnegative superharmonic function on $(M, g)$ if and only if $M$ is parabolic.

Let $K$ be a compact subset in $(M, g)$. We define the capacity $\operatorname{Cap}(K)$ by

$$
\operatorname{Cap}(K)=\inf \left\{\int_{M}|\nabla u|^{2} d m_{g}: u \in \operatorname{Lip}_{0}(M),\left.u\right|_{K} \equiv 1\right\}
$$

where $\operatorname{Lip}_{0}(M)$ is a set of all Lipschitz functions with compact support on $M$, and $m_{g}$ is the Riemannian measure associated to $g$. Then there is a nonconstant nonnegative superharmonic function on $M$ if and only if $\operatorname{Cap}(K)>0$ for some compact subset $K$, (see [7, Theorem 5.1] for Riemannian manifolds). It follows that the parabolicity of a Riemann surface $M$ can be characterized both in terms of capacity and superharmonic functions. By this reason, in the setting of Riemannian manifolds or metric measure spaces, one defines parabolicity either via capacity (see $[12,14,15,16]$ ) or via superharmonic functions (see [7] and also references therein). In this paper,

https://doi.org/10.54330/afm.112449

2020 Mathematics Subject Classification: Primary 31C05, 31C15, 31C45, 31E05.

Key words: Capacity, harmonic function, parabolicity, regular tree.

The author has been supported by the Academy of Finland (project No. 323960).

(c) 2022 The Finnish Mathematical Society 
we will consider $K$-regular trees and give the definition of parabolicity in terms of capacity.

Recently, analysis on $K$-regular trees has been under development, see [3, 21, $22,23,20,27]$. Let $G$ be a $K$-regular tree with a set of vertices $V$ and a set of edges $E$ for some $K \geq 1$. The union of $V$ and $E$ will be denoted by $X$. We abuse the notation and call $X$ a $K$-regular tree. We introduce a metric structure on $X$ by considering each edge of $X$ to be an isometric copy of the unit interval. Then the distance between two vertices is the number of edges needed to connect them and there is a unique geodesic that minimizes this number. Let us denote the root by 0 . If $x$ is a vertex, we define $|x|$ to be the distance between 0 and $x$. Since each edge is an isometric copy of the unit interval, we may extend this distance naturally to any $x$ belonging to an edge. We define $\partial X$ as the collection of all infinite geodesics starting at the root 0 . Then every $\xi \in \partial X$ corresponds to an infinite geodesic $[0, \xi)$ (in $X)$ that is an isometric copy of the interval $[0, \infty)$. Let $\mu$ and $\lambda:[0, \infty) \rightarrow(0, \infty)$ be locally integrable functions. Let $d|x|$ be the length element on $X$. We define a measure $\mu$ on $X$ by setting $d \mu(x)=\mu(|x|) d|x|$, and a metric $d$ on $X$ via $d s(x)=\lambda(|x|) d|x|$ by setting $d(y, z)=\int_{[y, z]} d s$ whenever $y, z \in X$ and $[y, z]$ is the unique geodesic between $y$ and $z$. Then $(X, d, \mu)$ is a metric measure space and hence one may define a Newtonian Sobolev space $N^{1, p}(X):=N^{1, p}(X, d, \mu)$ based on upper gradients $[10,24]$. As usual, $N_{0}^{1, p}(X)$ is the completion of the family of functions with compact support in $N^{1, p}(X)$, and $\dot{N}_{0}^{1, p}(X)$ is the completion of the family of functions with compact support in $\dot{N}^{1, p}(X)$, the homogeneous version of $N^{1, p}(X)$. Let $\Omega$ be a subset of $X$. We denote by $N_{\text {loc }}^{1, p}(\Omega)$ the space of all functions $u \in L_{\text {loc }}^{p}(\Omega)$ that have an upper gradient in $L_{\mathrm{loc}}^{p}(\Omega)$, where $L_{\mathrm{loc}}^{p}(\Omega)$ is the space of all measurable functions that are $p$-integrable on any compact subset of $\Omega$. See Section 2 for the precise definitions.

Let $1<p<\infty$ and $O$ be a subset of $X$. We define the $p$-capacity of $O$, denoted $\operatorname{Cap}_{p}(O)$, by setting

$$
\operatorname{Cap}_{p}(O)=\inf \left\{\int_{X} g_{u}^{p} d \mu:\left.u\right|_{O} \equiv 1, u \in N_{0}^{1, p}(X)\right\}
$$

where $g_{u}$ is the minimal upper gradient of $u$ as in Section 2.2. A $K$-regular tree $X$ is said to be $p$-parabolic if $\operatorname{Cap}_{p}(O)=0$ for all compact sets $O \subset X$; otherwise $X$ is p-hyperbolic.

Given $1<p<\infty$ and an open subset $\Omega \subseteq X$, we say that $u \in N_{\text {loc }}^{1, p}(\Omega)$ is a p-harmonic function (or a p-superharmonic function) on $\Omega$ if

$$
\int_{\operatorname{spt}(\varphi)} g_{u}^{p} d \mu \leq \int_{\operatorname{spt}(\varphi)} g_{u+\varphi}^{p} d \mu
$$

holds for all functions (or for all nonnegative functions) $\varphi \in N^{1, p}(\Omega)$ with compact support $\operatorname{spt}(\varphi) \subset \Omega$. We refer the interested readers to $[2,9,11]$ for a discussion on the $p$-capacity and $p$-(super)harmonic functions.

Since a $K$-regular tree $(X, d)$ is the quintessential Gromov hyperbolic space, it is then natural to ask for whether the parabolicity (or hyperbolicity) of $X$ can be characterized via $p$-(super)harmonic functions under some conditions on the measure $\mu$ only depending on the given metric $d$, and also ask for intrinsic conditions of $K$ regular trees that would characterize the parabolicity (or hyperbolicity). We refer the readers to [1, Chapter IV] for a discussion in the case of Riemann surfaces, and 
$[6,7,14,15,16]$ for a discussion in the setting of Riemannian manifolds, and [25, Section 6], [28] for a discussion on infinite networks.

In order to state our results, we introduce a notion from [21]. Let $1<p<\infty$. We set

$$
R_{p}(\lambda, \mu)=\int_{0}^{\infty} \lambda(t)^{\frac{p}{p-1}} \mu(t)^{\frac{1}{1-p}} K^{\frac{j(t)}{1-p}} d t
$$

where $j(t)$ is the smallest integer such that $j(t) \geq t$, and let $X^{n}=\{x \in X:|x| \leq n\}$ for each $n \in \mathbb{N}$. Since we work with a fixed pair $\lambda, \mu$, we will usually write $R_{p}(\lambda, \mu)$ simply as $R_{p}$ when no confusion can arise. In what follows, we additionally assume that $\lambda^{p} \mu^{-1} \in L_{\text {loc }}^{1 /(p-1)}([0, \infty))$ to make sure that the finiteness of $R_{p}$ is a condition at infinity.

The first result of our paper is a characterization of parabolicity of $K$-regular trees.

Theorem 1.1. Let $1<p<\infty$ and $X$ be a $K$-regular tree with metric $d$ and measure $\mu$ as above, with $K \geq 1$. Then $(X, d, \mu)$ is p-parabolic if and only if any one of the following conditions is fulfilled:

1. $R_{p}(\lambda, \mu)=\infty$.

2. $\operatorname{Cap}_{p}\left(X^{n}\right)=0$ for all $n \in \mathbb{N} \cup\{0\}$.

3. $\operatorname{Cap}_{p}\left(X^{n}\right)=0$ for some $n \in \mathbb{N} \cup\{0\}$.

In Section 2.1, we will show that the compactness on a $K$-regular tree $X$ with respect to our metric $d$ and with respect to the graph metric are equivalent. Since each compact set in $(X, d)$ is contained in some $n$-level set $X^{n}$ that is an analog of a ball with respect to graph metric, parabolicity of $X$ can be characterized by the zero $p$-capacity of some/ all $n$-level sets $X^{n}$.

In [21, Theorem 1.3], the condition $R_{p}(\lambda, \mu)=\infty$ gives a characterization of the existence of boundary trace operators and for density properties for $\dot{N}^{1, p}(X)$. Hence parabolicity of $K$-regular trees can be characterized in terms of boundary trace operators and density properties. Combining Theorem 1.1 and [21, Theorem 1.3 and Theorem 3.5], we obtain the following corollary.

Corollary 1.2. Let $1<p<\infty$ and $X$ be a $K$-regular tree with metric $d$ and measure $\mu$ as above, with $K \geq 1$. Then $(X, d, \mu)$ is p-parabolic if and only if any one of the following conditions is fulfilled:

1. There exists $u \in \dot{N}^{1, p}(X)$ such that

$$
\lim _{[0, \xi) \ni x \rightarrow \xi} u(x)=\infty
$$

for all $\xi \in \partial X$.

2. $\dot{N}_{0}^{1, p}(X)=\dot{N}^{1, p}(X)$.

It is well known, see for instance the survey paper [15], that the volume growth condition

$$
\int_{1}^{\infty}\left(\frac{t}{V(B(0, t))}\right)^{\frac{1}{p-1}} d t=\infty
$$

is a sufficient condition to guarantee parabolicity of Riemannian manifolds. Here $V(B(0, t))$ is the volume of the ball with radius $t$ and center at a fixed point 0 . However, this condition is far from being necessary in general, as shown by a counterexample due to Holopainen [14] and to Varopoulos [26] in the case $p=2$. Our condition $R_{p}(\lambda, \mu)=\infty$ is an analog of this volume growth condition. Example 3.8 in 
Section 3 shows that there exists a $K$-regular tree with a distance $d$ and a "non-radial" measure $\mu$ such that $R_{p}(\lambda, \mu)=\infty$ but $X$ is $p$-hyperbolic.

Let $1<p<\infty$ and let $\operatorname{int}\left(X^{n}\right):=\{x \in X:|x|<n\}$ for $n \in \mathbb{N}$. We say that $\left(\operatorname{int}\left(X^{n}\right), d, \mu\right)$ is doubling and supports a p-Poincaré inequality if there exist constants $C_{1} \geq 1, C_{2}>0$ only depending on $n$ such that for all balls $B(x, 2 r) \subset$ $\operatorname{int}\left(X^{n}\right)$,

$$
\mu(B(x, 2 r)) \leq C_{1} \mu(B(x, r))
$$

and for all balls $B(x, r) \subset \operatorname{int}\left(X^{n}\right)$,

$$
f_{B(x, r)}\left|u-u_{B(x, r)}\right| d \mu \leq C_{2} r\left(f_{B(x, r)} g^{p} d \mu\right)^{\frac{1}{p}}
$$

whenever $u$ is a measurable function on $B(x, r)$ and $g$ is an upper gradient of $u$, where $u_{B(x, r)}:=f_{B(x, r)} u d \mu=\frac{1}{\mu(B(x, r))} \int_{B(x, r)} u d \mu$. The validity of a $p$-Poincaré inequality for $X$ has very recently been characterized via a Muckenhoupt-type condition under a doubling condition on $(X, d, \mu)$, see [23] for more information.

Our second result deals with a characterization of parabolicity in terms of $p$ (super)- harmonic functions.

Theorem 1.3. Let $1<p<\infty$ and $X$ be a $K$-regular tree with metric $d$ and measure $\mu$ as above, with $K \geq 2$. Assume additionally that $\left(\operatorname{int}\left(X^{n}\right), d, \mu\right)$ is doubling and supports a $p$-Poincaré inequality for each $n \in \mathbb{N}$. Then $(X, d, \mu)$ is p-parabolic if and only if any one of the following conditions is fulfilled:

1. Every nonnegative $p$-superharmonic function $u$ on $X$ is constant.

2. Every nonnegative $p$-harmonic function $u$ on $X$ is constant.

3. Every bounded $p$-harmonic function $u$ on $X$ is constant.

4. Every bounded $p$-harmonic function $u$ on $X$ with $\int_{X} g_{u}^{p} d \mu<\infty$ is constant.

Let us close the introduction with some comments on Theorem 1.3. According to a version of Theorem 1.3 in the setting of Riemannian manifolds from $[12,13,17]$ we have that $1 . \Rightarrow 2 . \Rightarrow 3 . \Rightarrow 4$. However 3 . does not imply 2 . in general. Our condition that ( $\left.\operatorname{int}\left(X^{n}\right), d, \mu\right)$ is doubling and supports a $p$-Poincaré inequality for each $n \in \mathbb{N}$ is equivalent to $\mu$ being a locally doubling measure supporting a local $p$-Poincaré inequality on $(X, d)$.

Theorem 1.3 is not empty in the sense that there exist both $p$-parabolic and $p$-hyperbolic $K$-regular trees that are doubling and support a $p$-Poincaré inequality, see Example 3.9 in Section 3 for more details.

The motivation for our paper comes from classification problems of spaces. By the survey papers $[4,7]$, the development of potential theory in the setting of metric measure spaces leads to a classification of spaces as either $p$-parabolic or not. This dichotomy can be seen as a non-linear analog of the recurrence or transience dichotomy in the theory of Brownian motion. This classification is helpful in the development of a quasiconformal uniformization theory, or for a deeper understanding of the links between the geometry of hyperbolic spaces and the analysis on their boundaries at infinity.

The paper is organized as follows. In Section 2, we introduce $K$-regular trees, Newtonian spaces, and $p$-(super)harmonic functions on our trees. In Section 3, we give the proofs of Theorem 1.1 and Theorem 1.3.

Throughout this paper, the letter $C$ (sometimes with a subscript) will denote positive constants that usually depend only on the space and may change at different 
occurrences; if $C$ depends on $a, b, \ldots$, we write $C=C(a, b, \ldots)$. For any function $f \in L_{\mathrm{loc}}^{1}(X)$ and any measurable subset $A \subset X$, let $f_{A} f d \mu$ stand for $\frac{1}{\mu(A)} \int_{A} f d \mu$.

\section{Preliminaries}

2.1. Regular trees. A graph $G$ is a pair $(V, E)$, where $V$ is a set of vertices and $E$ is a set of edges. We call a pair of vertices $x, y \in V$ neighbors if $x$ is connected to $y$ by an edge. The degree of a vertex is the number of its neighbors. The graph structure gives rise to a natural connectivity structure. A tree $G$ is a connected graph without cycles. A graph (or tree) is made into a metric graph by considering each edge as a geodesic of length one.

We call a tree $G$ a rooted tree if it has a distinguished vertex called the root, which we will denote by 0 . The neighbors of a vertex $x \in V$ are of two types: the neighbors that are closer to the root are called parents of $x$ and all other neighbors are called children of $x$. Each vertex has a unique parent, except for the root itself that has none.

We say that a tree is $K$-regular if it is a rooted tree such that each vertex has exactly $K$ children for some integer $K \geq 1$. Then all vertices except the root of a $K$-regular tree have degree $K+1$, and the root has degree $K$.

Let $G$ be a $K$-regular tree with a set of vertices $V$ and a set of edges $E$ for some integer $K \geq 1$. For simplicity of notation, we let $X=V \cup E$ and call it a $K$-regular tree. For $x \in X$, let $|x|$ be the distance from the root 0 to $x$, that is, the length of the geodesic from 0 to $x$, where the length of every edge is 1 and we consider each edge to be an isometric copy of the unit interval. The geodesic connecting two points $x, y \in X$ is denoted by $[x, y]$. Throughout this paper, we denote $X^{n}:=\{x \in X:|x| \leq n\}$ and $\operatorname{int}\left(X^{n}\right):=\{x \in X:|x|<n\}$ for each $n \in \mathbb{N}$.

On our $K$-regular tree $X$, we define a measure $\mu$ and a metric $d$ via $d s$ by setting

$$
d \mu(x)=\mu(|x|) d|x|, \quad d s(x)=\lambda(|x|) d|x|,
$$

where $\lambda, \mu:[0, \infty) \rightarrow(0, \infty)$ are fixed with $\lambda, \mu \in L_{\text {loc }}^{1}([0, \infty))$. Here $d|x|$ is the measure which gives each edge Lebesgue measure 1 , as we consider each edge to be an isometric copy of the unit interval and the vertices are the end points of this interval. Hence for any two points $z, y \in X$, the distance between them is

$$
d(z, y)=\int_{[z, y]} d s(x)=\int_{[z, y]} \lambda(|x|) d|x|
$$

where $[z, y]$ is the unique geodesic from $z$ to $y$ in $X$.

We abuse the notation and let $\mu(x)$ and $\lambda(x)$ denote $\mu(|x|)$ and $\lambda(|x|)$, respectively, for any $x \in X$, if there is no danger of confusion. We denote by $d_{E}$ the graph metric on $X$. Then for any two points $z, y \in X$,

$$
d_{E}(z, y)=\int_{[z, y]} d|x|
$$

is the graph distance between $z$ and $y$ where $[z, y]$ is the unique geodesic from $z$ to $y$.

Theorem 2.1. The identity mapping $\operatorname{Id}_{X}:\left(X, d_{E}\right) \rightarrow(X, d)$ is a homeomorphism.

Proof. Let us first prove that the identity mapping $f:\left(X, d_{E}\right) \rightarrow(X, d), f(x)=x$ if $x \in X$, is continuous. Let $B_{d}(x, r)$ be an arbitrary open ball with center $x$ and radius $r>0$ in $(X, d)$. Recall that $\lambda:[0, \infty) \rightarrow(0, \infty)$ is a locally integrable function. 
Hence $\lambda$ is an integrable function on $[a, b]$ whenever $[a, b]$ is a compact interval with $|x| \in(a, b)$ if $x \neq 0$, or $|x|=a$ if $x=0$ where 0 is the root of $X$. Then

$$
F(h):=\int_{a}^{h} \lambda(t) d t
$$

is absolutely continuous on $[a, b]$. It follows that there exists $\delta_{r}>0$ only depending on $x, r$ such that

$$
\begin{cases}\int_{|x|+\delta_{r}}^{|x|+\delta_{r}} \lambda(t) d t<\frac{r}{2} & \text { if }|x| \in(a, b), x \neq 0 \\ \int_{|x|}^{|x|+\delta_{r}} \lambda(t) d t<\frac{r}{2} & \text { if }|x|=0 .\end{cases}
$$

The open ball with center $x$ and radius $\delta_{r}$ in $\left(X, d_{E}\right)$ is denoted by $B_{d_{E}}\left(x, \delta_{r}\right)$. For any $y \in B_{d_{E}}\left(x, \delta_{r}\right)$, we have that $[x, y] \subset[x, \bar{x}] \cup[\bar{x}, y]$ where $\bar{x} \in[0, x]$ with $d_{E}(x, \bar{x})=\delta_{r}$. Then the above estimate gives that

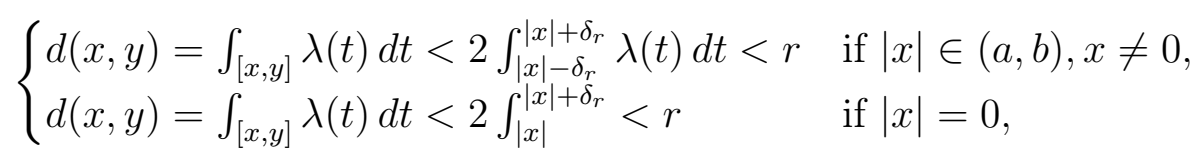

and hence $y \in B_{d}(x, r)$. As $B_{d}(x, r)$ is arbitrary, we obtain that for any open ball $B_{d}(x, r)$ there exists $\delta_{r}>0$ only depending on $x, r$ such that $B_{d_{E}}\left(x, \delta_{r}\right) \subset B_{d}(x, r)$. Thus

$$
\text { the identity mapping } f:\left(X, d_{E}\right) \rightarrow(X, d) \text { is continuous. }
$$

Next, we claim that also the identity mapping $g:(X, d) \rightarrow\left(X, d_{E}\right), g(x)=x$ if $x \in X$, is continuous. Let $B_{d_{E}}\left(x, r^{\prime}\right)$ be an arbitrary open ball with center $x$ and radius $r^{\prime}>0$ in $\left(X, d_{E}\right)$. We set

$$
\delta_{r^{\prime}}=\min \left\{\int_{|x|-r^{\prime} / 3}^{|x|} \lambda(t) d t, \int_{|x|}^{|x|+r^{\prime} / 3} \lambda(t) d t\right\} .
$$

Then $\delta_{r^{\prime}}>0$ since $\lambda>0$. We denote by $B_{d}\left(x, \delta_{r^{\prime}}\right)$ the open ball with center $x$ and radius $\delta_{r^{\prime}}$ in $(X, d)$. For any $y \in B_{d}\left(x, \delta_{r^{\prime}}\right)$, we have that

$$
\int_{[x, y]} \lambda(t) d t=d(x, y)<\delta_{r^{\prime}}
$$

It follows from (2.2) and (2.3) that $|z| \in\left[|x|-r^{\prime} / 3,|x|+r^{\prime} / 3\right]$ for any $z \in[x, y]$, and hence $d_{E}(x, z)<r^{\prime}$ for any $z \in[x, y]$. In particular, $d_{E}(x, y)<r^{\prime}$ for any $y \in B_{d}\left(x, \delta_{r^{\prime}}\right)$. Then $B_{d}\left(x, \delta_{r^{\prime}}\right) \subset B_{d_{E}}\left(x, r^{\prime}\right)$ for any $B_{d_{E}}\left(x, r^{\prime}\right)$. Therefore

$$
\text { the identity mapping } g:(X, d) \rightarrow\left(X, d_{E}\right) \text { is continuous. }
$$

We conclude from $(2.1)$ and $(2.4)$ that $\operatorname{Id}_{X}:\left(X, d_{E}\right) \rightarrow(X, d)$ is a homeomorphism. The claim follows.

We note that $X^{n}$ is compact in $\left(X, d_{E}\right)$ for each $n \in \mathbb{N}$ because it is a union of finitely many compact edges. Furthermore, any compact set in $\left(X, d_{E}\right)$ is contained in $X^{n}$ for some $n$ since any compact set in $\left(X, d_{E}\right)$ is bounded. Since compactness is preserved under homeomorphisms, we have the following corollaries.

Corollary 2.2. Let $O$ be an arbitrary compact set in $(X, d)$. Then $O \subset X^{n}$ for some $n \in \mathbb{N}$.

Corollary 2.3. Let $n \in \mathbb{N}$. Then $X^{n}$ is compact in $(X, d)$, and $\operatorname{int}\left(X^{n}\right)$ is open in $(X, d)$. 
Corollary 2.4. $(X, d, \mu)$ is a connected, locally compact, and non-compact metric measure space.

2.2. Newtonian spaces. Let $1<p<\infty$ and $X$ be a $K$-regular tree with metric $d$ and measure $\mu$ as in Section 2.1. Let $u \in L_{\text {loc }}^{1}(X)$. We say that a Borel function $g: X \rightarrow[0, \infty]$ is an upper gradient of $u$ if

$$
|u(y)-u(z)| \leq \int_{\gamma} g d s
$$

whenever $y, z \in X$ and $\gamma$ is the geodesic from $y$ to $z$. In the setting of our tree, any rectifiable curve with end points $z$ and $y$ contains the geodesic connecting $z$ and $y$, and therefore the upper gradient defined above is equivalent to the definition which requires that (2.5) holds for all rectifiable curves with end points $z$ and $y$. In $[8,11]$, the notion of a $p$-weak upper gradient is given. A Borel function $g: X \rightarrow[0, \infty]$ is called a $p$-weak upper gradient of $u$ if $(2.5)$ holds on $p$-a.e. curve. Here we say that a property holds for $p$-a.e. curve if it fails only for a curve family $\Gamma$ with zero $p$-modulus, i.e., there is a Borel nonnegative function $\rho \in L^{p}(X)$ such that $\int_{\gamma} \rho d s=\infty$ for any curve $\gamma \in \Gamma$. We refer to $[8,11]$ for more information about $p$-weak upper gradients.

The notion of upper gradients is due to Heinonen and Koskela [10], we refer interested readers to $[2,8,11,24]$ for a more detailed discussion on upper gradients. The following lemma of Fuglede shows that a converging sequence in $L^{p}$ has a subsequence that converges with respect to $p$-a.e. curve (see [11, Section 5.2]).

Lemma 2.5. (Fuglede's lemma) Let $\left\{g_{n}\right\}_{n=1}^{\infty}$ be a sequence of Borel nonnegative functions that converges to $g$ in $L^{p}(X)$. Then there is a subsequence $\left\{g_{n_{k}}\right\}_{k=1}^{\infty}$ such that

$$
\lim _{k \rightarrow \infty} \int_{\gamma}\left|g_{n_{k}}-g\right| d s=0
$$

for $p$-a.e. curve $\gamma$ in $X$.

The following useful results are from [11, Section 2.3 and Section 2.4] or [2, Section 6.1].

Theorem 2.6. Every bounded sequence $\left\{u_{n}\right\}_{n=1}^{\infty}$ in a reflexive normed space $\left(V,|\cdot|_{V}\right)$ has a weakly convergent subsequence $\left\{u_{n_{k}}\right\}_{k=1}^{\infty}$. Moreover, there exists $u \in V$ such that $u_{n_{k}} \rightarrow u$ weakly in $V$ as $k \rightarrow \infty$ and

$$
|u|_{V} \leq \liminf _{k \rightarrow \infty}\left|u_{n_{k}}\right|_{V}
$$

Lemma 2.7. (Mazur's lemma) Let $\left\{u_{n}\right\}_{n=1}^{\infty}$ be a sequence in a normed space $V$ converging weakly to an element $u \in V$. Then there exists a sequence $\bar{v}_{k}$ of convex combinations

$$
\bar{v}_{k}=\sum_{i=k}^{N_{k}} \lambda_{i, k} u_{i}, \quad \sum_{i=k}^{N_{k}} \lambda_{i, k}=1, \quad \lambda_{i, k} \geq 0
$$

converging to $v$ in the norm.

The Newtonian space $N^{1, p}(X), 1<p<\infty$, is defined as the collection of all the functions $u$ with finite $N^{1, p}$-norm

$$
\|u\|_{N^{1, p}(X)}:=\|u\|_{L^{p}(X)}+\inf _{g}\|g\|_{L^{p}(X)}
$$

where the infimum is taken over all upper gradients of $u$. We denote by $g_{u}$ the minimal upper gradient, which is unique up to measure zero and which is minimal in the sense that if $g \in L^{p}(X)$ is any upper gradient of $u$ then $g_{u} \leq g$ a.e. We refer to 
[8, Theorem 7.16] for proofs of the existence and uniqueness of such a minimal upper gradient.

If $u \in N^{1, p}(X)$, then it is continuous by (2.5) under the assumption $\frac{\lambda^{p}}{\mu} \in$ $L_{\text {loc }}^{\frac{1}{p-1}}([0, \infty))$ and it has a minimal $p$-weak upper gradient, see [21, Section 2]. More precisely, by [21, Proposition 2.2] the empty family is the only curve family with zero $p$-modulus, and hence any $p$-weak upper gradient is actually an upper gradient here and the conclusion of Lemma 2.5 holds for every curve $\gamma$. Moreover, it follows from [8, Definition 7.2 and Lemma 7.6] that any function $u \in L_{\mathrm{loc}}^{1}(X)$ with an upper gradient $0 \leq g \in L^{p}(X)$ is locally absolutely continuous, for example, absolutely continuous on each edge. The classical derivative $u^{\prime}$ of this locally absolutely continuous function is a minimal upper gradient in the sense that $g_{u}=\left|u^{\prime}(x)\right| / \lambda(x)$ when $u$ is parametrized in the natural way.

We define the homogeneous Newtonian space $\dot{N}^{1, p}(X), 1<p<\infty$, the collection of all the continuous functions $u$ that have an upper gradient $0 \leq g \in L^{p}(X)$, for which the homogeneous $\dot{N}^{1, p}$-norm of $u$ defined as

$$
\|u\|_{\dot{N}^{1, p}(X)}:=|u(0)|+\inf _{g}\|g\|_{L^{p}(X)}
$$

is finite. Here 0 is the root of our $K$-regular tree $X$ and the infimum is taken over all upper gradients of $u$.

The completion of the family of functions with compact support in $N^{1, p}(X)$ (or $\dot{N}^{1, p}(X)$ ) is denoted by $N_{0}^{1, p}(X)$ (or $\dot{N}_{0}^{1, p}(X)$ ). We denote by $N_{\text {loc }}^{1, p}(X)$ the space of all functions $u \in L_{\mathrm{loc}}^{p}(X)$ that have an upper gradient in $L_{\mathrm{loc}}^{p}(X)$, where $L_{\mathrm{loc}}^{p}(X)$ is the space of all measurable functions that are $p$-integrable on any compact subset of $X$. Especially, since each $X^{n}$ is compact in $(X, d)$ by Corollary 2.3 , we conclude that each $u \in N_{\text {loc }}^{1, p}(X)$ is both continuous and bounded on each $X^{n}$.

2.3. $\boldsymbol{p}$-(super)harmonic functions. Let $1<p<\infty$ and $X$ be a $K$-regular tree with metric $d$ and measure $\mu$ as in Section 2.1. For an open subset $\Omega$ of $X$, a function $u \in N_{\text {loc }}^{1, p}(\Omega)$ is said to be a $p$-harmonic function on $\Omega$ if

$$
\int_{\operatorname{spt}(\varphi)} g_{u}^{p} d \mu \leq \int_{\operatorname{spt}(\varphi)} g_{u+\varphi}^{p} d \mu
$$

holds for all functions $\varphi \in N^{1, p}(\Omega)$ with compact $\operatorname{support} \operatorname{spt}(\varphi) \subset \Omega$. We say that a function $u \in N_{\text {loc }}^{1, p}(\Omega)$ is a $p$-superharmonic function if (2.6) holds for all nonnegative functions $\varphi \in N^{1, p}(\Omega)$ with compact support $\operatorname{spt}(\varphi) \subset \Omega$.

We have a characterization of $p$-harmonic functions on $X$, see [2, Lemma 7.11].

Theorem 2.8. A function $u$ is a p-harmonic function on $X$ if and only if $u$ is $p$-harmonic on $\operatorname{int}\left(X^{n}\right)$ for all $n \in \mathbb{N}$.

By the stability properties of $p$-superharmonic functions (superminimizers) in general metric measure spaces (see for instance [2, Theorem 7.25]), since $\operatorname{int}\left(X^{n}\right)$ is open for each $n \in \mathbb{N}$ (see Corollary 2.3), we obtain the following results in our setting.

Theorem 2.9. Let $n \in \mathbb{N}$. If $\left\{u_{i}\right\}_{i \geq n}$ is a sequence of $p$-harmonic functions on $\operatorname{int}\left(X^{n}\right)$ which converges locally uniformly to $u \operatorname{in} \operatorname{int}\left(X^{n}\right)$, then $u$ is $p$-harmonic on $\operatorname{int}\left(X^{n}\right)$.

Let $1<p<\infty$ and $n \in \mathbb{N}$. Then $\left(\operatorname{int}\left(X^{n}\right), d, \mu\right)$ is said to be doubling and to support a $p$-Poincaré inequality if there exist constants $C_{1} \geq 1, C_{2}>0$ only 
depending on $n$ such that for all balls $B(x, 2 r) \subset \operatorname{int}\left(X^{n}\right)$,

$$
\mu(B(x, 2 r)) \leq C_{1} \mu(B(x, r))
$$

and for all balls $B(x, r) \subset \operatorname{int}\left(X^{n}\right)$,

$$
f_{B(x, r)}\left|u-u_{B(x, r)}\right| d \mu \leq C_{2} r\left(f_{B(x, r)} g^{p} d \mu\right)^{\frac{1}{p}}
$$

whenever $u$ is a measurable function on $B(x, r)$ and $g$ is an upper gradient of $u$. Recall that if $\left(\operatorname{int}\left(X^{n}\right), d, \mu\right)$ is doubling and supports a $p$-Poincaré inequality, then $N^{1, p}\left(\operatorname{int}\left(X^{n}\right), d, \mu\right)$ is a reflexive space (see [5, Theorem 4.48]).

Combining Proposition 3.9 and Theorem 5.4 in [19], we obtain the local Hölder continuity of $p$-harmonic functions on $\operatorname{int}\left(X^{n}\right)$ for each $n \in \mathbb{N}$.

Theorem 2.10. Let $n \in \mathbb{N}$. Assume that $\left(\operatorname{int}\left(X^{n}\right), d, \mu\right)$ is doubling and supports a $p$-Poincaré inequality. Then every $p$-harmonic function $u$ on $\operatorname{int}\left(X^{n}\right)$ is locally $\alpha$-Hölder continuous for some $0<\alpha \leq 1$.

\section{Proofs of Theorem 1.1 and Theorem 1.3}

In this section, if we do not specifically mention, we always assume that $1<$ $p<\infty$ and that $X$ is a $K$-regular tree with metric $d$ and measure $\mu$ as in Section 2.1.

Lemma 3.1. $X$ is p-parabolic if and only if $\operatorname{Cap}_{p}\left(X^{n}\right)=0$ for all $n \in \mathbb{N} \cup\{0\}$.

Proof. Let $X$ be $p$-parabolic. By Corollary 2.3, we have that $X^{n}$ is compact in $(X, d)$ for all $n \in \mathbb{N}$ and hence

$$
\operatorname{Cap}_{p}\left(X^{n}\right)=0
$$

for all $n \in \mathbb{N}$. This also holds for all $n \in \mathbb{N} \cup\{0\}$ because $\operatorname{Cap}_{p}\left(X^{0}\right) \leq \operatorname{Cap}_{p}\left(X^{n}\right)$. Conversely, suppose that

$$
\operatorname{Cap}_{p}\left(X^{n}\right)=0
$$

for all $n \in \mathbb{N} \cup\{0\}$. Let $O$ be an arbitrary compact set in $(X, d)$. Then $O \subset X^{n}$ for some $n \in \mathbb{N}$ by Corollary 2.2, and so that $\operatorname{Cap}_{p}(O) \leq \operatorname{Cap}_{p}\left(X^{n}\right)$. Combining this with (3.1) yields $\operatorname{Cap}_{p}(O)=0$. Since $O$ is arbitrary, we conclude that $X$ is $p$-parabolic. The proof is complete.

Lemma 3.2. Let $n \in \mathbb{N} \cup\{0\}$ be arbitrary. Then $R_{p}=\infty$ if and only if $\operatorname{Cap}_{p}\left(X^{n}\right)=0$.

Proof. Suppose that $R_{p}=\infty$. We first claim that $\operatorname{Cap}_{p}\left(X^{n}\right)=0$. Let us define a sequence $\left\{u_{k}\right\}_{k=n+1}^{\infty}$ by setting

$$
u_{k}(x)= \begin{cases}1 & \text { if } x \in X^{n}, \\ 1-\frac{\int_{n}^{|x|} \lambda^{\frac{p}{p-1}}(t) \mu^{\frac{1}{1-p}}(t) K^{\frac{j(t)}{1-p}} d t}{\int_{n}^{k} \lambda^{\frac{p}{p-1}}(t) \mu^{\frac{1}{1-p}}(t) K^{\frac{j(t)}{1-p}} d t} & \text { if } x \in X^{k} \backslash X^{n} \\ 0 & \text { otherwise. }\end{cases}
$$

Then

$$
g_{k}(x)=\frac{\lambda^{\frac{1}{p-1}}(x) \mu^{\frac{1}{1-p}}(x) K^{\frac{j(x)}{1-p}}}{\int_{n}^{k} \lambda^{\frac{p}{p-1}}(t) \mu^{\frac{1}{1-p}}(t) K^{\frac{j(t)}{1-p}} d t} \chi_{X^{k} \backslash X^{n}}(x)
$$


is an upper gradient of $u_{k}$. Next, a direct computation reveals that

$$
\int_{X} g_{u_{k}}^{p} d \mu \leq \int_{X} g_{k}^{p} d \mu=\frac{1}{\left(\int_{n}^{k} \lambda^{\frac{p}{p-1}}(t) \mu^{\frac{1}{1-p}}(t) K^{\frac{j(t)}{1-p}} d t\right)^{p-1}}<\infty
$$

for all $k \geq n+1$. Since $R_{p}=\infty$ and $\mu\left(X^{k}\right)<\infty$ for each $k \in \mathbb{N}$, it follows from (3.2)-(3.3) that $u_{k} \in N_{0}^{1, p}(X)$ with $\left.u_{k}\right|_{X^{n}} \equiv 1$ and that

$$
\lim _{k \rightarrow \infty} \int_{X} g_{u_{k}}^{p} d \mu=0
$$

We thus get $\operatorname{Cap}_{p}\left(X^{n}\right)=0$. Conversely, suppose that $\operatorname{Cap}_{p}\left(X^{n}\right)=0$. Then there exists a sequence $\left\{u_{k}\right\}_{k=1}^{\infty}$ in $N_{0}^{1, p}(X)$ with $\left.u_{k}\right|_{X^{n}} \equiv 1$ such that

$$
\lim _{k \rightarrow \infty} \int_{X} g_{u_{k}}^{p} d \mu=0
$$

Set $v_{k}:=u_{k}-1$. Then $g_{u_{k}}=g_{v_{k}}$. Combining this with $(3.4)$ and $u_{k}(0)=1$ yields

$$
\left\|u_{k}-1\right\|_{\dot{N}^{1, p}(X)}^{p}=\left\|v_{k}\right\|_{\dot{N}^{1, p}(X)}^{p}=\int_{X} g_{v_{k}}^{p} d \mu \rightarrow 0, \quad \text { as } k \rightarrow \infty .
$$

Therefore $u_{k} \rightarrow 1$ in $\dot{N}^{1, p}(X)$ with $u_{k} \in N_{0}^{1, p}(X)$, and hence $1 \in \dot{N}_{0}^{1, p}(X)$. Recall that $R_{p}=\infty$ is equivalent to $1 \in \dot{N}_{0}^{1, p}(X)$ by [21, Theorem 1.3 and Corollary 4.2]. Thus $R_{p}=\infty$ which completes the proof.

Lemma 3.3. Let $X$ be $p$-parabolic. Then every nonnegative $p$-superharmonic function $u$ on $X$ is constant.

Proof. Let $u \in N_{\text {loc }}^{1, p}(X)$ be an arbitrary nonnegative $p$-superharmonic function on $X$. We claim that $u$ is constant. Indeed, let $n_{0} \in \mathbb{N}$ be arbitrary. We denote

$$
M:=\|u\|_{L^{\infty}\left(X^{\left.n_{0}\right)}\right.} .
$$

Then $M<\infty$, since $u \in N_{\text {loc }}^{1, p}(X)$ is bounded on $X^{n}$ for each $n \in \mathbb{N}$, see the end of Section 2.2. By Lemma 3.1, we have $\operatorname{Cap}_{p}\left(X^{n_{0}}\right)=0$, and hence that there is a sequence $\left\{1_{n}\right\}_{n=1}^{\infty}$ in $N_{0}^{1, p}(X)$ with $\left.1_{n}\right|_{X^{n_{0}}} \equiv 1$ such that

$$
\lim _{n \rightarrow \infty} \int_{X} g_{1_{n}}^{p} d \mu=0 .
$$

Without loss of generality we assume that each $\operatorname{spt}\left(1_{n}\right)$ is compact. We define a sequence $\left\{\varphi_{n}\right\}_{n=1}^{\infty}$ by setting

$$
\varphi_{n}(x)=\max \left\{M \cdot 1_{n}(x), u(x)\right\}-u(x)
$$

for each $n \in \mathbb{N}$ and for all $x \in X$. Then

$$
\operatorname{spt}\left(\varphi_{n}\right) \subset \operatorname{spt}\left(1_{n}\right)
$$

for all $n \in \mathbb{N}$. We have that $0 \leq \varphi_{n} \in N^{1, p}(X)$ with compact support $\operatorname{spt}\left(\varphi_{n}\right)$, because (3.6) holds and $\operatorname{spt}\left(1_{n}\right)$ is compact. Since $u$ is $p$-superharmonic on $X$, it follows that

$$
\int_{\operatorname{spt}\left(\varphi_{n}\right)} g_{u}^{p} d \mu \leq \int_{\operatorname{spt}\left(\varphi_{n}\right)} g_{u+\varphi_{n}}^{p} d \mu
$$

for all $n \in \mathbb{N}$. As $u+\varphi_{n}=\max \left\{M \cdot 1_{n}, u\right\}$, we have that

$$
g_{u+\varphi_{n}}^{p}(x)=g_{M \cdot 1_{n}}^{p}(x) \chi_{\left\{x \in X: M \cdot 1_{n} \geq u\right\}}(x)+g_{u}^{p}(x) \chi_{\left\{x \in X: u>M \cdot 1_{n}\right\}}(x)
$$


for all $x \in X$. According to $M=\|u\|_{L^{\infty}\left(X^{n_{0}}\right)},\left.1_{n}\right|_{X^{n_{0}}} \equiv 1$, it follows that $u(x) \leq$ $M \cdot 1_{n}(x)$ and $M \cdot 1_{n}(x) \equiv M$ for all $x \in X^{n_{0}}$. Thanks to (3.6), we have that for all $x \in \operatorname{spt}\left(1_{n}\right)$,

$$
\chi_{\left\{x \in X: u>M \cdot 1_{n}\right\}}(x) \leq \chi_{\left\{x \in \operatorname{spt}\left(1_{n}\right) \backslash X^{n_{0}}\right\}}(x) \text { and } g_{M \cdot 1_{n}}=M \cdot g_{1_{n}} \chi_{\left\{x \in \operatorname{spt}\left(1_{n}\right) \backslash X^{n_{0}}\right\}} .
$$

Substituting (3.9) into (3.8) and combining with $\chi_{\left\{x \in X: M \cdot 1_{n} \geq u\right\}} \leq 1$ yields

$$
g_{u+\varphi_{n}}^{p}(x) \leq M^{p} \cdot g_{1_{n}}^{p}(x) \chi_{\left\{x \in \operatorname{spt}\left(1_{n}\right) \backslash X^{n_{0}}\right\}}(x)+g_{u}^{p}(x) \chi_{\left\{x \in \operatorname{spt}\left(1_{n}\right) \backslash X^{n_{0}}\right\}}(x)
$$

for all $x \in \operatorname{spt}\left(1_{n}\right)$. By (3.6), the above inequality holds for all $x \in \operatorname{spt}\left(\varphi_{n}\right)$. Then (3.7) gives that

$$
\int_{\operatorname{spt}\left(\varphi_{n}\right)} g_{u}^{p} d \mu \leq M^{p} \int_{\operatorname{spt}\left(\varphi_{n}\right) \backslash X^{n_{0}}} g_{1_{n}}^{p} d \mu+\int_{\operatorname{spt}\left(\varphi_{n}\right) \backslash X^{n_{0}}} g_{u}^{p} d \mu
$$

for all $n \in \mathbb{N}$. By $\int_{\operatorname{spt}\left(\varphi_{n}\right) \backslash X^{n_{0}}} g_{u}^{p} d \mu<\infty$, because $u \in N_{\text {loc }}^{1, p}(X)$ and $\operatorname{spt}\left(\varphi_{n}\right)$ is compact, subtracting $\int_{\operatorname{spt}\left(\varphi_{n}\right) \backslash X^{n_{0}}} g_{u}^{p} d \mu$ from both sides of (3.10) yields

$$
\int_{X^{n_{0}}} g_{u}^{p} d \mu \leq M^{p} \int_{\operatorname{spt}\left(\varphi_{n}\right) \backslash X^{n_{0}}} g_{1_{n}}^{p} d \mu \leq M^{p} \int_{X} g_{1_{n}}^{p} d \mu
$$

for all $n \in \mathbb{N}$. Letting $n \rightarrow \infty$, we conclude from (3.5) and (3.11) that $\int_{X^{n_{0}}} g_{u}^{p} d \mu=0$. Since $n_{0}$ is arbitrary, this implies that $u$ is constant, and the claim follows.

Remark 3.4. Assume that $f>0$ is a $p$-superharmonic function on $X$. Then

$$
\int_{X} f^{-p} g_{f}^{p} \varphi^{p} d \mu \leq\left(\frac{p}{p-1}\right)^{p} \int_{X} g_{\varphi}^{p} d \mu
$$

for all $\varphi \in N_{0}^{1, p}(X)$ with $0 \leq \varphi \leq 1$. This inequality (3.12) is often called a Caccioppoli-type inequality, see Section 3 in [18].

One can give an alternate proof for Lemma 3.3 via the Caccioppoli inequality (3.12). Indeed, let $u$ be an arbitrary nonnegative $p$-superharmonic function on $X$. Suppose that $X$ is $p$-parabolic. By Lemma 3.1, we have that $\operatorname{Cap}_{p}\left(X^{n}\right)=0$ for all $n \in \mathbb{N}$. Let $n \in \mathbb{N}$ be arbitrary. Then for any $\varepsilon>0$ there exists $u_{n, \varepsilon} \in N_{0}^{1, p}(X)$ with $0 \leq u_{n, \varepsilon} \leq 1$ and $\left.u_{n, \varepsilon}\right|_{X^{n}} \equiv 1$ such that

$$
\int_{X} g_{u_{n, \varepsilon}}^{p} d \mu \leq \operatorname{Cap}_{p}\left(X^{n}\right)+\varepsilon=\varepsilon .
$$

Applying the Caccioppoli inequality (3.12) for $f=u+1$ with $\varphi=u_{n, \varepsilon}$, yields

$$
\int_{X}(u+1)^{-p} g_{u+1}^{p} u_{n, \varepsilon}^{p} d \mu \leq\left(\frac{p}{p-1}\right)^{p} \int_{X} g_{u_{n, \varepsilon}}^{p} d \mu .
$$

Note that $g_{\log (u+1)}=(u+1)^{-1} g_{u+1}$ by [2, Theorem 2.16 or Proposition 2.17]. We combine this and (3.13)-(3.14) with $\left.u_{n, \varepsilon}\right|_{X^{n}} \equiv 1$ to obtain that

$$
\int_{X^{n}} g_{\log (u+1)}^{p} d \mu \leq\left(\frac{p}{p-1}\right)^{p} \varepsilon .
$$

Letting $\varepsilon \rightarrow 0$, this gives $g_{\log (u+1)}=0$ on $X^{n}$ and hence that $u$ is constant on $X^{n}$. Thus $u$ is constant on $X$ since $n \in \mathbb{N}$ is arbitrary.

Let $x_{0}$ be a closest vertex of the root 0 of a $K$-regular tree where $K \geq 2$. Then we set

$$
T_{x_{0}}:=\left\{y \in X: x_{0} \in[0, y]\right\} \quad \text { and } \quad T_{1}:=\left[0, x_{0}\right] \cup T_{x_{0}} .
$$


For any $n \in \mathbb{N}$, we denote

$$
E_{n}:=\left(X \backslash X^{n}\right) \cap T_{1} \text { and } F_{n}:=\left(X \backslash X^{n}\right) \backslash T_{1} .
$$

We define the $p$-capacity of the pair $\left(E_{n}, F_{n}\right)$, denoted $\operatorname{Cap}_{p}\left(E_{n}, F_{n}\right)$, by setting

$$
\operatorname{Cap}_{p}\left(E_{n}, F_{n}\right)=\inf \left\{\int_{X} g_{u}^{p} d \mu: u \in N_{\text {loc }}^{1, p}(X),\left.u\right|_{E_{n}} \equiv 1,\left.u\right|_{F_{n}} \equiv 0,0 \leq u \leq 1\right\} .
$$

The following lemma follows straightforwardly from the definition (3.15) of $p$-capacity.

Lemma 3.5. Let $X$ be a $K$-regular tree where $K \geq 2$ and let $1<p<\infty$. Then the sequence $\left\{\operatorname{Cap}_{p}\left(E_{n}, F_{n}\right)\right\}_{n=1}^{\infty}$ is non-increasing and there exists a constant $0<M_{1}<\infty$ such that

$$
\operatorname{Cap}_{p}\left(E_{n}, F_{n}\right) \leq M_{1}<\infty \text { for all } n \in \mathbb{N} .
$$

Proof. It is clear from (3.15) that $\left\{\operatorname{Cap}_{p}\left(E_{n}, F_{n}\right)\right\}_{n=1}^{\infty}$ is non-increasing and bounded from above by $\operatorname{Cap}_{p}\left(E_{1}, F_{1}\right)$. As the piecewise linear function $f$ with $\left.f\right|_{E_{1}} \equiv 1,\left.f\right|_{F_{1}} \equiv 0$ is admissible for computing $\operatorname{Cap}_{p}\left(E_{1}, F_{1}\right)$, the claim follows since

for all $n \in \mathbb{N}$.

$$
\operatorname{Cap}_{p}\left(E_{n}, F_{n}\right) \leq \operatorname{Cap}_{p}\left(E_{1}, F_{1}\right) \leq \int_{X} g_{f}^{p} d \mu \leq \frac{\mu\left(X^{1}\right)}{d\left(E_{1}, F_{1}\right)^{p}}<\infty
$$

Lemma 3.6. Let $X$ be a $p$-hyperbolic $K$-regular tree where $K \geq 2$ and $1<$ $p<\infty$. Suppose that, for each $n \in \mathbb{N}$, (int $\left.\left(X^{n}\right), d, \mu\right)$ is doubling and supports a p-Poincaré inequality. Then there exist a constant $0<M_{2}<\infty$ and a sequence $\left\{u_{n}\right\}_{n=1}^{\infty}$ in $N_{\text {loc }}^{1, p}(X)$ with $\left.u_{n}\right|_{E_{n}} \equiv 1,\left.u_{n}\right|_{F_{n}} \equiv 0,0 \leq u_{n} \leq 1$ and so that each $u_{n}$ is a nonconstant $p$-harmonic function on $\operatorname{int}\left(X^{n}\right)$ with

$$
0<M_{2} \leq \int_{X^{n}} g_{u_{n}}^{p} d \mu=\operatorname{Cap}_{p}\left(E_{n}, F_{n}\right)
$$

Proof. Let $n \in \mathbb{N}$. By the definition (3.15) of $\operatorname{Cap}_{p}\left(E_{n}, F_{n}\right)$, there exists a sequence $\left\{u_{n, m}\right\}_{m=1}^{\infty}$ in $N_{\text {loc }}^{1, p}(X)$ with $\left.u_{n, m}\right|_{E_{n}} \equiv 1,\left.u_{n, m}\right|_{F_{n}} \equiv 0,0 \leq u_{n, m} \leq 1$ such that

$$
\operatorname{Cap}_{p}\left(E_{n}, F_{n}\right) \leq \int_{X} g_{u_{n, m}}^{p} d \mu \leq \operatorname{Cap}_{p}\left(E_{n}, F_{n}\right)+\frac{1}{m}
$$

By Lemma 3.5, there is a constant $0<M_{1}<\infty$ such that

$$
\operatorname{Cap}_{p}\left(E_{n}, F_{n}\right)<M_{1}<\infty \text { for all } n \in \mathbb{N}
$$

and hence $\left\{g_{u_{n, m}}\right\}_{m=1}^{\infty}$ is bounded in $L^{p}(X)$. We have from $\mu\left(X^{n+1}\right)<\infty, 0 \leq$ $u_{n, m} \leq 1$ that $\left\{u_{n, m}\right\}_{m=1}^{\infty}$ is bounded in $L^{p}\left(X^{n+1}\right)$. Then $\left\{u_{n, m}\right\}_{m=1}^{\infty}$ is bounded in $N^{1, p}\left(X^{n+1}\right)$. We note that $N^{1, p}\left(\operatorname{int}\left(X^{n+1}\right)\right)$ is a reflexive space since $\left(\operatorname{int}\left(X^{n+1}\right), d, \mu\right)$ is doubling and supports a $p$-Poincaré inequality, see [5, Theorem 4.48]. Hence Theorem 2.6 gives that there is a subsequence $\left\{u_{n, m_{k}}\right\}_{k=1}^{\infty}$ which converges weakly to some $u_{n} \in N^{1, p}\left(\operatorname{int}\left(X^{n+1}\right)\right)$. By Mazur's Lemma 2.7, there is a sequence of convex combinations $f_{k}$ which converges to $u_{n}$ in $N^{1, p}\left(\operatorname{int}\left(X^{n+1}\right)\right)$ :

$$
f_{k}:=\sum_{i=k}^{N_{k}} a_{i, k} u_{n, i}
$$

where $a_{i, k} \geq 0, \sum_{i=k}^{N_{k}} a_{i, k}=1, u_{n, i} \in\left\{u_{n, m_{k}}\right\}_{k=1}^{\infty}$. We may assume that $f_{k}(x)$ converges pointwise to $u_{n}(x)$ as $k \rightarrow \infty$ on $\operatorname{int}\left(X^{n+1}\right)$. It is easy to see that $\left.u_{n}\right|_{\operatorname{int}\left(X^{n+1}\right) \cap E_{n}} \equiv 1$ 
and $\left.u_{n}\right|_{\operatorname{int}\left(X^{n+1}\right) \cap F_{n}} \equiv 0$ from (3.19) since $\left.u_{n, i}\right|_{\operatorname{int}\left(X^{n+1}\right) \cap E_{n}} \equiv 1,\left.u_{n, i}\right|_{\operatorname{int}\left(X^{n+1}\right) \cap F_{n}} \equiv 0$ for all $i$. We extend $u_{n}$ by setting $\left.u_{n}\right|_{E_{n} \backslash \operatorname{int}\left(X^{n+1}\right)} \equiv 1,\left.u_{n}\right|_{F_{n} \backslash \operatorname{int}\left(X^{n+1}\right)} \equiv 0$. Then

$$
u_{n} \in L_{\text {loc }}^{p}(X) \text { with }\left.u_{n}\right|_{E_{n}} \equiv 1,\left.u_{n}\right|_{F_{n}} \equiv 0,0 \leq u_{n} \leq 1 .
$$

Next, we have by the convexity of the function $t \mapsto t^{p}$ that

$$
\int_{X^{n}} g_{f_{k}}^{p} d \mu \leq \sum_{i=k}^{N_{k}} a_{i, k} \int_{X^{n}} g_{u_{n, i}}^{p} d \mu .
$$

By the triangle inequality, this gives

$$
\begin{aligned}
\left(\int_{X^{n}} g_{u_{n}}^{p} d \mu\right)^{\frac{1}{p}} & \leq\left(\int_{X^{n}} g_{f_{k}-u_{n}}^{p} d \mu\right)^{\frac{1}{p}}+\left(\int_{X^{n}} g_{f_{k}}^{p} d \mu\right)^{\frac{1}{p}} \\
& \leq\left(\int_{X^{n}} g_{f_{k}-u_{n}}^{p} d \mu\right)^{\frac{1}{p}}+\left(\sum_{i=k}^{N_{k}} a_{i, k} \int_{X^{n}} g_{u_{n, i}}^{p} d \mu\right)^{\frac{1}{p}} .
\end{aligned}
$$

According to (3.17) and $\sum_{i=k}^{N_{k}} a_{i, k}=1$,

$$
\begin{aligned}
\left(\sum_{i=k}^{N_{k}} a_{i, k} \int_{X^{n}} g_{u_{n, i}}^{p} d \mu\right)^{\frac{1}{p}} & \leq\left(\sum_{i=k}^{N_{k}} a_{i, k} \operatorname{Cap}_{p}\left(E_{n}, F_{n}\right)+\sum_{i=k}^{N_{k}} a_{i, k} \frac{1}{i}\right)^{\frac{1}{p}} \\
& \leq\left(\operatorname{Cap}_{p}\left(E_{n}, F_{n}\right)+\frac{1}{k}\right)^{\frac{1}{p}} .
\end{aligned}
$$

Substituting (3.22) into (3.21) and combining with $f_{k} \rightarrow u_{n}$ in $N^{1, p}\left(\operatorname{int}\left(X^{n+1}\right)\right)$ as $k \rightarrow \infty$, we obtain that

$$
\left(\int_{X^{n}} g_{u_{n}}^{p} d \mu\right)^{\frac{1}{p}} \leq\left(\int_{X^{n}} g_{f_{k}-u_{n}}^{p} d \mu\right)^{\frac{1}{p}}+\left(\operatorname{Cap}_{p}\left(E_{n}, F_{n}\right)+\frac{1}{k}\right)^{\frac{1}{p}} \rightarrow \operatorname{Cap}_{p}\left(E_{n}, F_{n}\right)^{\frac{1}{p}}
$$

as $k \rightarrow \infty$. Then the above estimate gives via (3.18) and $\left.u_{n}\right|_{F_{n}} \equiv 0,\left.u_{n}\right|_{E_{n}} \equiv 1$ that $g_{u_{n}} \in L^{p}(X)$. Combining this with (3.20) yields $u_{n} \in N_{\text {loc }}^{1, p}(X)$ with $\left.u_{n}\right|_{E_{n}} \equiv 1$, $\left.u_{n}\right|_{F_{n}} \equiv 0,0 \leq u_{n} \leq 1$ and hence $u_{n}$ is admissible for computing the capacity $\operatorname{Cap}_{p}\left(E_{n}, F_{n}\right)$. It follows from this and the above estimate that

$$
\int_{X^{n}} g_{u_{n}}^{p} d \mu=\operatorname{Cap}_{p}\left(E_{n}, F_{n}\right) \text { for all } n \in \mathbb{N} .
$$

We conclude from (3.18), (3.20), (3.23) that there are a constant $0<M_{1}<\infty$ and a function $u_{n} \in N_{\text {loc }}^{1, p}(X)$ with $\left.u_{n}\right|_{E_{n}} \equiv 1,\left.u_{n}\right|_{F_{n}} \equiv 0$, and $0 \leq u_{n} \leq 1$ such that

$$
\int_{X^{n}} g_{u_{n}}^{p} d \mu=\operatorname{Cap}_{p}\left(E_{n}, F_{n}\right) \leq M_{1}<\infty \text { for all } n \in \mathbb{N} \text {. }
$$

We now prove that there is a constant $0<M_{2}<\infty$ such that

$$
0<M_{2} \leq \int_{X^{n}} g_{u_{n}}^{p} d \mu=\operatorname{Cap}_{p}\left(E_{n}, F_{n}\right) \text { for all } n \in \mathbb{N} .
$$

Let $n \in \mathbb{N}$ and $u_{n}$ be as in (3.24). If $u_{n}(0)<1 / 2$, we define $v:=\max \{0, \min \{1,2(1-$ $\left.\left.u_{n}\right)\right\}$. Then $v \in N_{\text {loc }}^{1, p}(X)$ with $v(0)=1,\left.v\right|_{E_{n}} \equiv 0$, and hence

$$
\operatorname{Cap}_{p}\left(\{0\}, E_{n}\right) \leq \int_{X} g_{v}^{p} d \mu \leq 2^{p} \int_{X^{n}} g_{u_{n}}^{p} d \mu=2^{p} \operatorname{Cap}_{p}\left(E_{n}, F_{n}\right) .
$$


If $u_{n}(0) \geq 1 / 2$, we define $w:=\max \left\{0, \min \left\{1,2 u_{n}\right\}\right\}$. Then $w \in N_{\text {loc }}^{1, p}(X)$ with $w(0)=1,\left.w\right|_{F_{n}} \equiv 0$, and hence

$$
\operatorname{Cap}_{p}\left(\{0\}, F_{n}\right) \leq \int_{X} g_{w}^{p} d \mu \leq 2^{p} \int_{X^{n}} g_{u_{n}}^{p} d \mu=2^{p} \operatorname{Cap}_{p}\left(E_{n}, F_{n}\right) .
$$

Combining the above estimates for each $n \in \mathbb{N}$, we obtain that

$\inf _{n \in \mathbb{N}} \min \left\{\operatorname{Cap}_{p}\left(\{0\}, E_{n}\right), \operatorname{Cap}_{p}\left(\{0\}, F_{n}\right)\right\} \leq 2^{p} \int_{X^{n}} g_{u_{n}}^{p} d \mu=2^{p} \operatorname{Cap}_{p}\left(E_{n}, F_{n}\right)$ for all $n \in \mathbb{N}$.

To obtain (3.25), we show that

$$
M_{2}:=\inf _{n \in \mathbb{N}} \min \left\{\operatorname{Cap}_{p}\left(\{0\}, E_{n}\right), \operatorname{Cap}_{p}\left(\{0\}, F_{n}\right)\right\}>0 .
$$

Applying Lemma 3.1 and Lemma 3.2 for the subtrees $T_{1}$ and $X \backslash T_{1}$, we obtain that

$$
T_{1} \text { is } p \text {-hyperbolic if and only if } R_{p}^{T_{1}}:=\frac{1}{K} R_{p}<\infty
$$

and

$$
X \backslash T_{1} \text { is } p \text {-hyperbolic if and only if } R_{p}^{X \backslash T_{1}}:=\frac{K-1}{K} R_{p}<\infty .
$$

Notice that if $X$ is $p$-hyperbolic, then both $T_{1}$ and $X \backslash T_{1}$ are $p$-hyperbolic since $R_{p}<\infty$ implies finiteness of $R_{p}^{T_{1}}$ and $R_{p}^{X \backslash T_{1}}$. By Lemma 3.2 with the $p$-hyperbolicity of $T_{1}$ and $X \backslash T_{1}$, it follows that

$$
\operatorname{Cap}_{p}\left(\{0\}, E_{n}\right) \geq \operatorname{Cap}_{p}^{T_{1}}(\{0\}):=\inf \left\{\int_{T_{1}} g_{u}^{p} d \mu: u \in N_{0}^{1, p}\left(T_{1}\right), u(0)=1\right\}>0
$$

and

$\operatorname{Cap}_{p}\left(\{0\}, F_{n}\right) \geq \operatorname{Cap}_{p}^{X \backslash T_{1}}(\{0\}):=\inf \left\{\int_{X \backslash T_{1}} g_{u}^{p} d \mu: u \in N_{0}^{1, p}\left(X \backslash T_{1}\right), u(0)=1\right\}>0$ for each $n \in \mathbb{N}$. Hence (3.26) holds.

Finally, we only need to show that $u_{n}$ is a $p$-harmonic function on $\operatorname{int}\left(X^{n}\right)$. Let $\varphi$ be an arbitrary element of $N^{1, p}\left(\operatorname{int}\left(X^{n}\right)\right)$ with compact support $\operatorname{spt}(\varphi) \subset \operatorname{int}\left(X^{n}\right)$. By choosing $v=\max \left\{0, \min \left\{1, u_{n}+\varphi\right\}\right\}$ we have that $v \in N^{1, p}\left(\operatorname{int}\left(X^{n}\right)\right)$ with $\left.v\right|_{E_{n}} \equiv 1,\left.v\right|_{F_{n}} \equiv 0,0 \leq v \leq 1$ because $\operatorname{spt}(\varphi) \subset \operatorname{int}\left(X^{n}\right)$ and because (3.20) holds. It follows from the definition (3.15) of $\operatorname{Cap}_{p}\left(E_{n}, F_{n}\right)$ that

$$
\operatorname{Cap}_{p}\left(E_{n}, F_{n}\right) \leq \int_{X^{n}} g_{v}^{p} d \mu \leq \int_{X^{n}} g_{u_{n}+\varphi}^{p} d \mu .
$$

Combining this with (3.23), we obtain that

$$
\int_{X^{n}} g_{u_{n}}^{p} d \mu \leq \int_{X^{n}} g_{u_{n}+\varphi}^{p} d \mu
$$

for all $\varphi \in N^{1, p}\left(\operatorname{int}\left(X^{n}\right)\right)$ with compact support $\operatorname{spt}(\varphi) \subset \operatorname{int}\left(X^{n}\right)$. Hence $u_{n}$ is $p$-harmonic on $\operatorname{int}\left(X^{n}\right)$, and the claim follows.

Lemma 3.7. Let $X$ be a $p$-hyperbolic $K$-regular tree where $K \geq 2$ and $1<p<$ $\infty$. Suppose that $\left(\operatorname{int}\left(X^{n}\right), d, \mu\right)$ is doubling and supports a $p$-Poincaré inequality for each $n \in \mathbb{N}$. Then there exists a nonconstant nonnegative bounded $p$-harmonic function $u$ on $X$ with $0<\int_{X} g_{u}^{p} d \mu<\infty$. 
Proof. We first prove that there exists a nonnegative bounded $p$-harmonic function $u$ on $X$. Towards this, Lemma 3.5 and Lemma 3.6 give that there exist constants $0<M_{2} \leq M_{1}<\infty$ and a sequence $\left\{u_{n}\right\}_{n=1}^{\infty}$ in $N_{\text {loc }}^{1, p}(X)$ with $\left.u_{n}\right|_{E_{n}} \equiv 1,\left.u_{n}\right|_{F_{n}} \equiv 0$, $0 \leq u_{n} \leq 1$ such that $u_{n}$ is a nonconstant $p$-harmonic function on $\operatorname{int}\left(X^{n}\right)$ and

$$
0<M_{2} \leq \int_{X^{n}} g_{u_{n}}^{p} d \mu=\operatorname{Cap}_{p}\left(E_{n}, F_{n}\right) \leq M_{1}<\infty \text { for all } n \in \mathbb{N} .
$$

Let $n_{0} \in \mathbb{N}$ be arbitrary. It follows from the local Hölder continuity of $p$-harmonic functions (see Theorem 2.10) that $\left\{u_{n}\right\}_{n \geq n_{0}}$ is equibounded and locally equicontinuous on $\operatorname{int}\left(X^{n_{0}+1}\right)$. By the Arzelà-Ascoli theorem, there exists a subsequence, still denoted $\left\{u_{n}\right\}_{n \geq n_{0}}$, that converges to $u$ uniformly on $X^{n_{0}}$ as $n \rightarrow \infty$. Since $n_{0}$ is arbitrary, by uniqueness of locally uniform convergence, we may assume that $u_{n}$ converges to $u$ locally uniformly in $X$ as $n \rightarrow \infty$. Then $\left\{u_{i}\right\}_{i \geq n}$ is a sequence of $p$-harmonic functions on int $\left(X^{n}\right)$ which converge uniformly to $u$ in $\operatorname{int}\left(X^{n}\right)$ for each $n$, and hence by Theorem 2.9 and Theorem 2.8 we obtain that $u$ is a $p$-harmonic function on $X$. Thus $u$ is a nonnegative bounded $p$-harmonic function on $X$ since $0 \leq u_{n} \leq 1$.

We next show that

$$
0<\int_{X} g_{u}^{p} d \mu<\infty
$$

It follows from (3.27) that $\left\{g_{u_{n}}\right\}_{n=1}^{\infty}$ is a bounded sequence in the reflexive space $L^{p}(X)$, and hence Theorem 2.6 and Mazur's Lemma 2.7 give that there exists $g \in$ $L^{p}(X)$ and a convex combination sequence $\bar{g}_{n}=\sum_{i=n}^{N_{n}} a_{i, n} g_{u_{i}}$ with $a_{i, n} \geq 0, \sum_{i=n}^{N_{n}} a_{i, n}$ $=1$ such that $\bar{g}_{n} \rightarrow g$ in $L^{p}(X)$ as $n \rightarrow \infty$. By Fuglede's Lemma 2.5, we obtain that there is a subsequence, still denoted $\bar{g}_{n}$, such that

$$
\lim _{n \rightarrow \infty} \int_{[x, y]} \bar{g}_{n} d s=\int_{[x, y]} g d s
$$

for every curve $[x, y]$. Note that $\bar{g}_{n}=\sum_{i=n}^{N_{n}} a_{i, n} g_{u_{i}}$ is an upper gradient of $\bar{u}_{n}=$ $\sum_{i=n}^{N_{n}} a_{i, n} u_{i}$ and $\bar{u}_{n}$ converges to $u$ locally uniformly in $X$ as $n \rightarrow \infty$. Hence

$$
|u(x)-u(y)|=\lim _{n \rightarrow \infty}\left|\bar{u}_{n}(x)-\bar{u}_{n}(y)\right| \leq \lim _{n \rightarrow \infty} \int_{[x, y]} \bar{g}_{n} d s=\int_{[x, y]} g d s
$$

for every curve $[x, y]$. Then $g$ is an upper gradient of $u$ and hence $g_{u} \leq g$ a.e. Combining this with $\bar{g}_{n} \rightarrow g$ in $L^{p}(X)$ as $n \rightarrow \infty$ and with the convexity of the function $t \mapsto t^{p}$ yields

$$
\int_{X} g_{u}^{p} d \mu \leq \int_{X} g^{p} d \mu=\lim _{n \rightarrow \infty} \int_{X} \bar{g}_{n}^{p} d \mu \leq \lim _{n \rightarrow \infty} \sum_{i=n}^{N_{n}} a_{i, n} \int_{X} g_{u_{i}}^{p} d \mu .
$$

Notice that $\left\{\operatorname{Cap}_{p}\left(E_{n}, F_{n}\right)\right\}_{n=1}^{\infty}$ is a nonincreasing sequence by Lemma 3.5. Hence we have by $\left.u_{i}\right|_{F_{i}} \equiv 0,\left.u_{i}\right|_{E_{i}} \equiv 1$ and $(3.27)$ that

$$
\begin{aligned}
\lim _{n \rightarrow \infty} \sum_{i=n}^{N_{n}} a_{i, n} \int_{X} g_{u_{i}}^{p} d \mu & =\lim _{n \rightarrow \infty} \sum_{i=n}^{N_{n}} a_{i, n} \int_{X^{i}} g_{u_{i}}^{p} d \mu=\lim _{n \rightarrow \infty} \sum_{i=n}^{N_{n}} a_{i, n} \operatorname{Cap}_{p}\left(E_{i}, F_{i}\right) \\
& \leq \lim _{n \rightarrow \infty} \sum_{i=n}^{N_{n}} a_{i, n} \operatorname{Cap}_{p}\left(E_{n}, F_{n}\right)=\lim _{n \rightarrow \infty} \operatorname{Cap}_{p}\left(E_{n}, F_{n}\right)<\infty .
\end{aligned}
$$

Substituting the above estimate into (3.28) yields $\int_{X} g_{u}^{p} d \mu<\infty$. 
It remains to show that $\int_{X} g_{u}^{p} d \mu>0$. The preceding being understood, we argue by contradiction and assume that $\int_{X} g_{u}^{p} d \mu=0$. Then $u$ is constant on $X$. Recall that $\bar{g}_{n}=\sum_{i=n}^{N_{n}} a_{i, n} g_{u_{i}}$ with $a_{i, n} \geq 0, \sum_{i=n}^{N_{n}} a_{i, n}=1$ are such that $\bar{g}_{n} \rightarrow g$ in $L^{p}(X)$ as $n \rightarrow \infty$ and

$$
\lim _{n \rightarrow \infty} \int_{[x, y]} \bar{g}_{n} d s=\int_{[x, y]} g d s
$$

for every curve $[x, y]$. Moreover, $\bar{g}_{n}$ is an upper gradient of $\bar{u}_{n}=\sum_{i=n}^{N_{n}} a_{i, n} u_{i}$ and $\bar{u}_{n}$ is admissible for computing the capacity $\operatorname{Cap}_{p}\left(E_{N_{n}}, F_{N_{n}}\right)$, and so

$$
\int_{X} \bar{g}_{n}^{p} d \mu \geq \int_{X} g_{\bar{u}_{n}}^{p} d \mu \geq \operatorname{Cap}_{p}\left(E_{N_{n}}, F_{N_{n}}\right) .
$$

Combining this with (3.27) and using $\bar{g}_{n} \rightarrow g$ in $L^{p}(X)$ as $n \rightarrow \infty$ yields

$$
\int_{X} g^{p} d \mu>0
$$

Let $\varepsilon>0$ be arbitrary, and let $[x, y]$ be an arbitrary edge in $X$. Since $u_{n}$ converges to $u$ locally uniformly in $X$, there exist positive constants $N, r$ only depending on $x, y$ such that for all $n \geq N$,

$$
\sup _{t \in B(x, r)}\left|u_{n}(t)-u(t)\right|<\varepsilon, \sup _{t \in B(y, r)}\left|u_{n}(t)-u(t)\right|<\varepsilon
$$

where $B(x, r), B(y, r)$ are balls with centers $x, y$ and radius $r$, respectively. Since $u$ is constant, the above estimates yield that for all $n \geq N$,

$$
\left|u_{n}(x)-u_{n}(y)\right| \leq\left|u_{n}(x)-u(x)\right|+\left|u(y)-u_{n}(y)\right|<2 \varepsilon .
$$

By Section 2.2, $u_{n}$ is absolutely continuous on $[x, y]$ and $g_{u_{n}}(z)=\left|u_{n}^{\prime}(z)\right| / \lambda(z)$ for $z \in[x, y]$. By the strong maximum principle (see for instance [19, Corollary 6.5]), $u_{n}$ is a monotone function on $[x, y]$. Hence

$$
\left|u_{n}(x)-u_{n}(y)\right|=\int_{[x, y]}\left|u_{n}^{\prime}(z)\right| d z .
$$

Since the minimal upper gradient $g_{u_{n}}$ of $u_{n}$ satisfies $g_{u_{n}}(x)=\left|u_{n}^{\prime}(x)\right| / \lambda(z)$, we conclude that

$$
\int_{[x, y]} g_{u_{n}} d s \leq 2 \varepsilon
$$

for all $n \geq N$. By (3.29), it follows that

$$
\int_{[x, y]} g d s=\lim _{n \rightarrow \infty} \int_{[x, y]} \bar{g}_{n} d s=\lim _{n \rightarrow \infty} \sum_{i=n}^{N_{n}} a_{i, n} \int_{[x, y]} g_{u_{i}} d s<2 \varepsilon .
$$

Letting $\varepsilon \rightarrow 0$, we obtain that $g=0$ a.e. on $[x, y]$. As $[x, y]$ is arbitrary, we conclude that $g=0$ a.e. which contradicts (3.30). This completes the proof.

Proof of Theorem 1.1. $X$ is $p$-parabolic $\Leftrightarrow 2$. is given by Lemma 3.1.

1 . $\Leftrightarrow 2$. $\Leftrightarrow 3$. is given by Lemma 3.2.

Proof of Theorem 1.3. $X$ is $p$-parabolic $\Rightarrow 1$. is given by Lemma 3.3.

1. $\Rightarrow 2$. is trivial.

2. $\Rightarrow 3$. : Let $u$ be a bounded $p$-harmonic function on $X$. Then there exists a constant $C>0$ such that $u+C$ is a nonnegative $p$-harmonic function on $X$. Hence $u+C$ is constant by the assumption and so $u$ is constant.

3 . $\Rightarrow 4$. is trivial. 
4. $\Rightarrow X$ is $p$-parabolic is given by Lemma 3.7 .

The following example shows that Theorem 1.1 is not true for general metrics and measures.

Example 3.8. Let $1<p<\infty$. There exists a $p$-hyperbolic $K$-regular tree $X$ with a distance and a "non-radial" measure such that $R_{p}=\infty$.

Let us begin with some notation. For simplicity, let $X$ be a dyadic tree (which means $K=2$ ). Then the root 0 of our tree has two closest vertices, denoted $v_{1}$ and $v_{2}$. We denote

$$
T_{1}=\left[0, v_{1}\right] \cup\left\{x \in X: v_{1} \in[0, x]\right\} \text { and } T_{2}=\left[0, v_{2}\right] \cup\left\{x \in X: v_{2} \in[0, x]\right\} .
$$

Note that the union of $T_{1}$ and $T_{2}$ is our tree. Suppose $\lambda_{i}, \mu_{i}:[0, \infty) \rightarrow(0, \infty)$ satisfy $\lambda_{i}, \mu_{i} \in L_{\mathrm{loc}}^{1}([0, \infty))$, for $i=1,2$. We introduce a measure $\mu$ and a metric $d$ via $d s$ by setting

$$
d \mu(x)=\mu_{i}(|x|) d|x|, \quad d s(x)=\lambda_{i}(|x|) d|x|,
$$

for all $x \in T_{i}$, for $i=1,2$. To obtain what we desire, we choose $\lambda_{1} \equiv \mu_{1} \equiv 1$ and $\lambda_{2} \equiv 1, \mu_{2}(x)=2^{-j(x)}$. Define a metric $d$ and a measure $\mu$ as above. Then

$$
\begin{aligned}
& \left.R_{p}\right|_{T_{1}}:=\frac{1}{2} \int_{0}^{\infty} \lambda_{1}^{\frac{p}{p-1}}(t) \mu_{1}^{\frac{1}{1-p}}(t) 2^{\frac{j(t)}{1-p}} d t<\infty, \\
& \left.R_{p}\right|_{T_{2}}:=\frac{1}{2} \int_{0}^{\infty} \lambda_{2}^{\frac{p}{p-1}}(t) \mu_{2}^{\frac{1}{1-p}}(t) 2^{\frac{j(t)}{1-p}} d t=\infty
\end{aligned}
$$

and $R_{p}=\left.R_{p}\right|_{T_{1}}+\left.R_{p}\right|_{T_{2}}=\infty$. By Theorem 1.1 for the subtree $T_{1}$ with $\left.R_{p}\right|_{T_{1}}<\infty$, we obtain that $T_{1}$ is $p$-hyperbolic. Hence there exists a compact set $O$ in $T_{1}$ such that $\operatorname{Cap}_{p}^{T_{1}}(O)>0$, where

$$
\operatorname{Cap}_{p}^{T_{1}}(O):=\inf \left\{\int_{T_{1}} g_{u}^{p} d \mu:\left.u\right|_{O} \equiv 1, u \in N_{0}^{1, p}\left(T_{1}\right)\right\}
$$

Let $O$ be such a set. Then $O$ is bounded in $T_{1}$ by Corollary 2.2. Let $u \in N_{0}^{1, p}(X)$ be an arbitrary function with $\left.u\right|_{O} \equiv 1$. It follows from $u \in N_{0}^{1, p}(X)$ that there exists a sequence $u_{n} \in N^{1, p}(X)$ with compact support $\operatorname{spt}\left(u_{n}\right) \subset X$ such that $u_{n} \rightarrow u$ in $N^{1, p}(X)$ as $n \rightarrow \infty$. By Corollary 2.2, we may assume that $\operatorname{spt}\left(u_{n}\right) \subset X^{n}$ for each $n$. Hence $\operatorname{spt}\left(u_{n}\right) \cap T_{1} \subset X^{n} \cap T_{1}$, and so $\operatorname{spt}\left(u_{n}\right) \cap T_{1}$ is compact in $T_{1}$ because $\operatorname{spt}\left(u_{n}\right) \cap T_{1}$ is a closed set in $T_{1}$ and $X^{n} \cap T_{1}$ is compact in $T_{1}$. Then for each $n$, $u_{n} \in N^{1, p}\left(T_{1}\right)$ with compact support $\operatorname{spt}\left(u_{n}\right)$ and $u_{n} \rightarrow u$ in $N^{1, p}\left(T_{1}\right)$ as $n \rightarrow \infty$, and hence $u \in N_{0}^{1, p}\left(T_{1}\right)$ with $\left.u\right|_{O} \equiv 1$. Thus $u$ is admissible for computing $\operatorname{Cap}_{p}^{T_{1}}(O)$ and so

$$
\int_{X} g_{u}^{p} d \mu \geq \int_{T_{1}} g_{u}^{p} d \mu \geq \operatorname{Cap}_{p}^{T_{1}}(O)
$$

Since $u \in N_{0}^{1, p}(X)$ with $\left.u\right|_{O} \equiv 1$ is arbitrary, the above estimate gives

$$
\operatorname{Cap}_{p}(O) \geq \operatorname{Cap}_{p}^{T_{1}}(O) .
$$

Combining this with $\operatorname{Cap}_{p}^{T_{1}}(O)>0$, we have $\operatorname{Cap}_{p}(O)>0$. Thus $X$ is $p$-hyperbolic.

Example 3.9. Let $1<p<\infty$. There exist both $p$-hyperbolic and $p$-parabolic $K$-regular trees $(X, d, \mu)$ that are doubling and support a $p$-Poincaré inequality. 
We begin with the $p$-hyperbolic case. Let $\mu(t)=e^{-\beta j(t)}$ and $\lambda(t)=e^{-\varepsilon j(t)}$ with $\varepsilon, \beta>0$ and $\log K<\beta<\log K+\varepsilon p$. It is obvious that $\mu(X)<\infty$ and $R_{p}<\infty$. More precisely, since $\log K<\beta<\log K+\varepsilon p$ we have that

$$
\mu(X)=\int_{0}^{\infty} \mu(t) K^{j(t)} d t=\int_{0}^{\infty} e^{-(\beta-\log K) j(t)} d t<\infty
$$

and

$$
R_{p}=\int_{0}^{\infty} \lambda(t)^{\frac{p}{p-1}} \mu(t)^{\frac{1}{1-p}} K^{\frac{j(t)}{1-p}} d t=\int_{0}^{\infty} e^{\frac{(\beta-\log K-\varepsilon p) j(t)}{p-1}} d t<\infty .
$$

As $R_{p}<\infty$, by Theorem 1.1 , it follows that $(X, d, \mu)$ is a noncomplete $p$-hyperbolic metric measure space. By [3, Section 3 and Section 4] or [22, Section 2] for $\log K<\beta$, we obtain that $(X, d, \mu)$ is doubling and supports a 1-Poincaré inequality.

For the $p$-parabolic case, let $\mu(t)=e^{-\beta j(t)}$ and $\lambda(t)=e^{-\varepsilon j(t)}$ with $\varepsilon, \beta>0$ and $\beta=\log K+\varepsilon p$. It is easy to see that $(X, d, \mu)$ is a noncomplete $p$-parabolic $K$-regular tree that is doubling and supports a 1-Poincaré inequality.

Acknowledgement. The author thanks his advisor Professor Pekka Koskela for helpful discussions. The author also would like to thank Zhuang Wang for reading the manuscript and giving comments that helped to improve the paper. The author is extremely grateful to Professor Jana Björn and the referees for their comments, suggestions, and corrections.

\section{References}

[1] Ahlfors, L. V., and L. Sario: Riemann surfaces. - Princeton Math. Ser. 26, Princeton Univ. Press, 1960.

[2] BJörn, A., and J. BJÖRN: Nonlinear potential theory on metric spaces. - EMS Tracts Math. 17, Eur. Math. Soc. (EMS), Zürich, 2011.

[3] Björn, A., J. Buörn, J. T. Gill, and N. Shanmugalingam: Geometric analysis on Cantor sets and trees. - J. Reine Angew. Math. 725, 2017, 63-114.

[4] Bonk, M., L. Capogna, P. HajŁasz, N. Shanmugalingam, and J. Tyson: Analysis in metric spaces. - Notices Amer. Math. Soc. 67:2, 2020, 253-256.

[5] Cheeger, J.: Differentiability of Lipschitz functions on metric measure spaces. - Geom. Funct. Anal. 9:3, 1999, 428-517.

[6] Gol'dshtein, V., and M. Troyanov: The Kelvin-Nevanlinna-Royden criterion for $p$ parabolicity. - Math. Z. 232:4, 1999, 607-619.

[7] Grigor'yan, A.: Analytic and geometric background of recurrence and non-explosion of the Brownian motion on Riemannian manifolds. - Bull. Amer. Math. Soc. (N.S.) 36:2, 1999, 135249.

[8] HajŁAsz, P.: Sobolev spaces on metric-measure spaces. - In: Heat kernels and analysis on manifolds, graphs, and metric spaces (Paris, 2002), Contemp. Math. 338, Amer. Math. Soc., Providence, RI, 2003, 173-218.

[9] Heinonen, J., T. KilpeläInen, and O. Martio: Nonlinear potential theory of degenerate elliptic equations. - Dover Publications, Inc., Mineola, NY, 2006.

[10] Heinonen, J., and P. Koskela: Quasiconformal maps in metric spaces with controlled geometry. - Acta Math. 181:1, 1998, 1-61.

[11] Heinonen, J., P. Koskela, N. Shanmugalingam, and J. T. Tyson: Sobolev spaces on metric measure spaces. An approach based on upper gradients. - New Math. Monogr. 27, Cambridge Univ. Press, Cambridge, 2015.

[12] Holopainen, I.: Nonlinear potential theory and quasiregular mappings on Riemannian manifolds. - Ann. Acad. Sci. Fenn. Ser. A I Math. Diss. 74, 1990, 1-45. 
[13] Holopainen, I.: Positive solutions of quasilinear elliptic equations on Riemannian manifolds. - Proc. London Math. Soc. (3) 65:3, 1992, 651-672.

[14] Holopainen, I.: Volume growth, Green's functions, and parabolicity of ends. - Duke Math. J. 97:2, 1999, 319-346.

[15] Holopainen, I., and P. Koskela: Volume growth and parabolicity. - Proc. Amer. Math. Soc. 129:11, 2001, 3425-3435.

[16] Holopainen, I., S. Markvorsen, and V. Palmer: p-Capacity and p-hyperbolicity of submanifolds. - Rev. Mat. Iberoam. 25:2, 2009, 709-738.

[17] Holopainen, I., and S. Rickman: Classification of Riemannian manifolds in nonlinear potential theory. - Potential Anal. 2:1, 1993,37-66.

[18] Kinnunen, J., and O. Martio: Sobolev space properties of superharmonic functions on metric spaces. - Results Math. 44:1-2, 2003, 114-129.

[19] Kinnunen, J., and N. Shanmugalingam: Regularity of quasi-minimizers on metric spaces. - Manuscripta Math. 105:3, 2001, 401-423.

[20] Koskela, P., K. Nguyen, and Z. Wang: Trace operators on regular trees. - Anal. Geom. Metr. Spaces 8:1, 2020, 396-409.

[21] Koskela, P., K. Nguyen, and Z. Wang: Trace and density results on regular trees. Potential Anal. (to appear).

[22] Koskela, P., and Z. WANG: Dyadic norm Besov-type spaces as trace spaces on regular trees. - Potential Anal. 53:4, 2020, 1317-1346.

[23] Nguyen, K., and Z. Wang: Admissibility versus $A_{p}$-conditions on regular trees. - Anal. Geom. Metr. Spaces 8:1, 2020, 92-105.

[24] Shanmugalingam, N.: Newtonian spaces: an extension of Sobolev spaces to metric measure spaces. - Rev. Mat. Iberoamericana 16:2, 2000, 243-279.

[25] Troyanov, M.: Parabolicity of manifolds. - Siberian Adv. Math. 9:4, 1999, 125-150.

[26] Varopoulos, N. T.: Potential theory and diffusion on Riemannian manifolds. - In: Conference on harmonic analysis in honor of Antoni Zygmund, Vol. I, II (Chicago, Ill., 1981), Wadsworth Math. Ser., Wadsworth, Belmont, CA, 1983, 821-837.

[27] WANG, Z.: Characterization of trace spaces on regular trees via dyadic norms. - J. Math. Anal. Appl. 494:2, 2021, 124646, 1-21.

[28] Yamasaki, M.: Parabolic and hyperbolic infinite networks. - Hiroshima Math. J. 7:1, 1977, $135-146$.

Received 5 November 2020 • Accepted 12 March $2021 \bullet$ Published online 29 November 2021

Khanh Nguyen

University of Jyväskylä

Department of Mathematics and Statistics

P. O. Box 35

FI-40014 University of Jyväskylä

Finland

khanh.n.nguyen@jyu.fi 